SECTION 5. Innovative technologies in science.

Korneyev Andrey Mastislavovich candidate of technical Sciences, associate Professor, Lipetsk state technical University, Russia

\author{
Abdullakh Lutfi Salekh \\ postgraduate student \\ Lipetsk state technical University, Russia
}

\title{
AUTOMATED SYSTEM FOR THE DESIGN PROCESS AND THE GENERATION OF TECHNOLOGICAL INFORMATION.
}

This research aims at exploring Automated system for the design process and the generation of technological information. Generation of technological information is performed with the aim further statistical processing of other sub-system for optimal process, conditions and making mathematical models of technological processes.

Key words: automation; design; system; generation; the process.

\section{АВТОМАТИЗИРОВАННАЯ СИСТЕМА ПРОЕКТИРОВАНИЯ ТЕХНОЛОГИЧЕСКИХ ПРОЦЕССОВ И ГЕНЕРАЦИИ ТЕХНОЛОГИЧЕСКОЙ ИНФОРМАЦИИ}

Автоматизированная система проектирования технологических процессов и генерации технологической информации системь управления предприятием предназначена для автоматизачии проектирования технологических процуессов и генерации технологической информации. Генерация технологической информациии производится с цельюю ее дальнейшей статистической обработки другими подсистемами для получения оптимальных технологических режимов и составления математических моделей техпроцессов.

Ключевые слова: автоматизирование; проектирование; система; генерация; процесс.

Если представить структуру предприятия в виде следующей иерархии уровней:
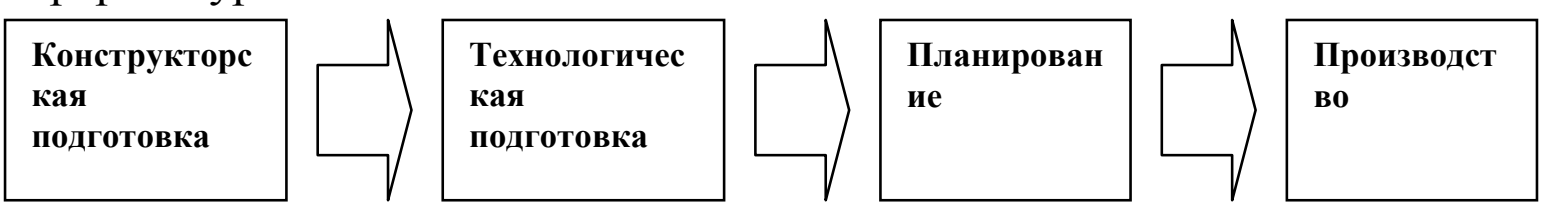
то подсистема будет работать на уровне технологической подготовки. Как видно из схемы уровень технологической подготовки граничит с уровнями конструкторской подготовки и планирования. С уровня конструкторской подготовки подсистема получает технологию или если выражаться в терминах нашей предметной области дерево операций. Результат работы системы, который она может передать другим подсистемам, будет представлять технологические цепочки и выборки сгенерированной технологической информации. Сгенерированная технологическая информация будет предназначаться для других подсистем находящихся на уровне технологической подготовки. Технологические цепочки будут передаваться на уровень планирования, для расчѐта потребностей производства в ресурсах, определения расходных коэффициентов, коэффициентов ресурсоѐмкости и анализа незавершѐнного производства. Подсистема не имеет строго иерархической структуры $\mathrm{c}$ чѐтко выраженными связями между автоматизируемыми функциями внутри самой подсистемы и связь между ними может осуществлять посредством внешних подсистем.

Так как система должна содержать большое количество справочной информации, то необходимо наличие следующих подсистем: подсистемы ведения справочника стандартов, сортамента, технологических режимов. Видно, что перечисленные подсистемы функционально близки друг к другу, так как они должны взаимодействовать с БД. Поэтому, возможно объединение данных подсистем в одну логическую структуру.

Получение технологических режимов возможно путѐм извлечения информации из стандартов или путѐм статистической обработки данных полученных из предыдущего опыта производства. Если технологические режимы извлечены из стандартов, то в систему они заносятся пользователем, если получены в результате статистической обработки, то внешней подсистемой.

Система должна уметь работать с деревом операций и генерировать технологические цепочки, поэтому необходимо наличие ещѐ двух подсистем. Подсистемы формирования дерева операций и подсистемы формирования цепочек агрегатов. Функционально данные подсистемы зависимы, и поэтому, необходимо наличие интерфейсов для доступа к экспортируемым функциям.

Подсистема формирования цепочек агрегатов должна передавать сгенерированные цепочки другим подсистемам. И соответственно данной подсистеме должен быть известен интерфейс взаимодействия с внешней подсистемой.

\section{Описание информационных функций по каждой подсистеме и решаемых в рамках каждой функции задач}




\section{1. Модуль формирования деревьев операций}

Осуществляет формирование дерева операций в диалоговом режиме с пользователем, либо производит выборку дерева из БД. Предоставляет информацию пользователю в графическом, удобном для восприятия виде.

Работа в диалоговом режиме производится в случае поступления на предприятие заказа, который требует нестандартного подхода к производству (требования заказчика не соответствуют ГОСТу либо другому стандарту), либо технология является новой не апробированной на предприятии. Сформированное дерево сохраняется в БД.

В случае если предприятие уже выполняло заказ на требуемую продукцию хотя бы раз, то соответствующее дерево операций уже находится в БД и в формировании нового нет необходимости. Осуществляется выборка дерева из БД (преобразование из машинного представления в графическое, визуальное).

Модуль осуществляет взаимодействие с модулем формирования цепочек агрегатов. Передаѐт ему сформированное дерево.

Время выполнения зависит от оператора, если формирование дерево операций происходит в автоматизированном режиме (вводится пользователем), либо от СУБД, если выборка дерева осуществляется из базы данных.

Результат работы отображается на экране в графическом виде и сохраняется в машинном виде в БД.

Точность работы зависит от оператора.

Прекращение работоспособности возможно либо в случае отказа технических средств, либо в случае отказа сервера БД.

\section{2. Модуль формирования цепочек агрегатов}

Получает на вход дерево операций. В зависимости от вида изделия, информация о котором извлекается из справочника сортамента, осуществляется проход дерева от корня до одного из его листов, соответствующего получению продукции требуемого вида. Передаѐт результаты во внешнюю среду.

Время работы зависит от количества уровней иерархии дерева операций, от вычислительной мощности технических средств, скорости работы внешних подсистем, осуществляющих определение оптимальных технологических режимов.

Результат проецируется на экран в графическом виде и сохраняется в БД.

Точность работы зависит от точности реализуемых алгоритмов.

Отказ возможен в случае отказа технических средств или отказа сервера БД. 


\section{3. Подсистема ведения справочной информации}

Даѐт возможность на понятном для пользователя -языке" (в терминах предметной области) осуществлять действия со справочной информацией (внесение новой информации, корректировка, поиск).

Время работы зависит от пользователя, скорости работы сервера БД, вычислительной мощности технических средств.

Результат проецируется на экран в виде таблиц и связных списков, а также сохраняется в БД.

Отказ возможен в случае отказа технических средств, либо в случае отказа сервера БД.

\section{4. Модуль генерации технологической информации}

Осуществляет генерацию технологической информации и соединение массивов технологических факторов. Передаѐт результат во внешнюю среду другим подсистемам.

Время работы зависит от вычислительной мощности технических средств и объѐмов входной и выходной информации.

Точность работы зависит от точности реализуемых алгоритмов.

Отказ возможен в случае отказа технических средств.

\section{Литература}

1. Зильбербург Л. И., Молочник В. И., Яблочников Е. И.

Информационные технологии в проектировании производстве. - СПб: Политехника, 2008. - 304 с.

2. Гафуров Х.Л., Гафуров Т.Х., Смирнов В.П. Системы автоматизированного проектирования: Учеб. пособие. - СПб.: Судостроение, 2006. -21-27 с.

3. Норенков, И. П. Основы автоматизированного проектирования / И. П. Норенков. - М. : Изд-во МГТУ, 2000. - 360 с. 